Revue des patrimoines

29 | 2016

Ensembles mobiliers, industriels, techniques.

Connaissance, protection, conservation, présentation au public

\title{
Francis Jourdain (1876-1958), un précurseur du mobilier contemporain
}

Francis Jourdain (1876-1958), a precursor of contemporary furniture design

\section{Sylvie Gonzalez}

\section{OpenEdition}

Journals

Édition électronique

URL : http://journals.openedition.org/insitu/12955

DOI : 10.4000/insitu. 12955

ISSN : $1630-7305$

Éditeur

Ministère de la Culture

Référence électronique

Sylvie Gonzalez, «Francis Jourdain (1876-1958), un précurseur du mobilier contemporain », In Situ [En ligne], 29 | 2016, mis en ligne le 13 juillet 2016, consulté le 14 novembre 2019. URL : http:// journals.openedition.org/insitu/12955; DOI : 10.4000/insitu.12955

Ce document a été généré automatiquement le 14 novembre 2019.

\section{(c) $(1) \Theta$}

In Situ Revues des patrimoines est mis à disposition selon les termes de la licence Creative Commons Attribution - Pas d'Utilisation Commerciale - Pas de Modification 4.0 International. 


\title{
Francis Jourdain (1876-1958), un précurseur du mobilier contemporain
}

Francis Jourdain (1876-1958), a precursor of contemporary furniture design

\author{
Sylvie Gonzalez
}

1 Francis Jourdain, ensemblier du début du $\mathrm{XX}^{\mathrm{e}}$ siècle, s'avère être l'un des précurseurs de l'aménagement intérieur. Son principe des «meubles interchangeables » créés en 1919, est repris aujourd'hui par une chaîne suédoise d'ameublement. Diffusée par la presse, en vente par correspondance, à la boutique ou sur commande, la production de Francis Jourdain veut s'adresser à tous, selon ses moyens.

\section{Un parcours moderne ${ }^{1}$}

2 Francis Jourdain naît le 2 novembre 1876 à Paris, dans une famille aisée de la bourgeoisie. Il a deux sœurs. Son père, Frantz Jourdain (1847-1935), d'origine belge, naturalisé français en 1870, est architecte, critique et grand amateur d'art, homme de lettres. Ernest Cognacq, homme d'affaire et fondateur avec son épouse Marie-Louise Jaÿ des grands magasins La Samaritaine, lui commande la construction des immeubles parisiens. Il fonde en 1903 le Salon d'automne et en est le président, ainsi que celui du Syndicat de la critique d'art. Le cercle intellectuel de la famille est ainsi très dense, composé des écrivains Alphonse Daudet (1840-1897), Émile Zola (1840-1902), les frères Goncourt, Edmond(1822-1896) et Jules (1830-1870), des peintres Claude Monet (1840-1926), Auguste Renoir (1841-1919), Eugène Carrière (1849-1906), Félix Vallotton (1865-1925) et Édouard Vuillard (1868-1940), du sculpteur Auguste Rodin (1840-1977), des architectes Georges Desvallières (1861-1950) et Hector Guimard (1867-1942).

3 Très jeune, Francis fréquente avec son ami Louis Rouart, fils du collectionneur, les expositions et les galeries (fig. 1). Il y rencontre d'autres artistes. Actif dans le mouvement anarchiste, il écrit des articles dans ses journaux. L'influence de son 
éducation, sa rencontre avec le décorateur Alexandre Charpentier, le décident à devenir ouvrier d'art. Il étudie le dessin chez Joseph Chéret, frère de Jules, l'affichiste, ami de son père, s'inscrit à l'académie Humbert et Gervex où enseigne Eugène Carrière. Il apprend le métier d'ornemaniste avec le peintre décorateur Adrian Karbowsky, collaborateur de Puvis de Chavannes. Il participe à la décoration de l'amphithéâtre de la Sorbonne auprès d'Albert Besnard et s'initie à la gravure dans l'atelier d'Eugène Delâtre. Il fait son apprentissage chez le maître verrier Clamens à Angers et est reçu premier au concours d'ouvrier d'art. De retour à Paris, il fréquente le cercle artistique d'Alexandre Charpentier. Il y rencontre ses amis artisans, le céramiste Jean Dampt, le joaillier Henri Nocq, l'architecte et dessinateur Charles Plumet, les ébénistes Félix Aubert et Tony Selmersheim qui auront une grande influence sur son travail.

Figure 1

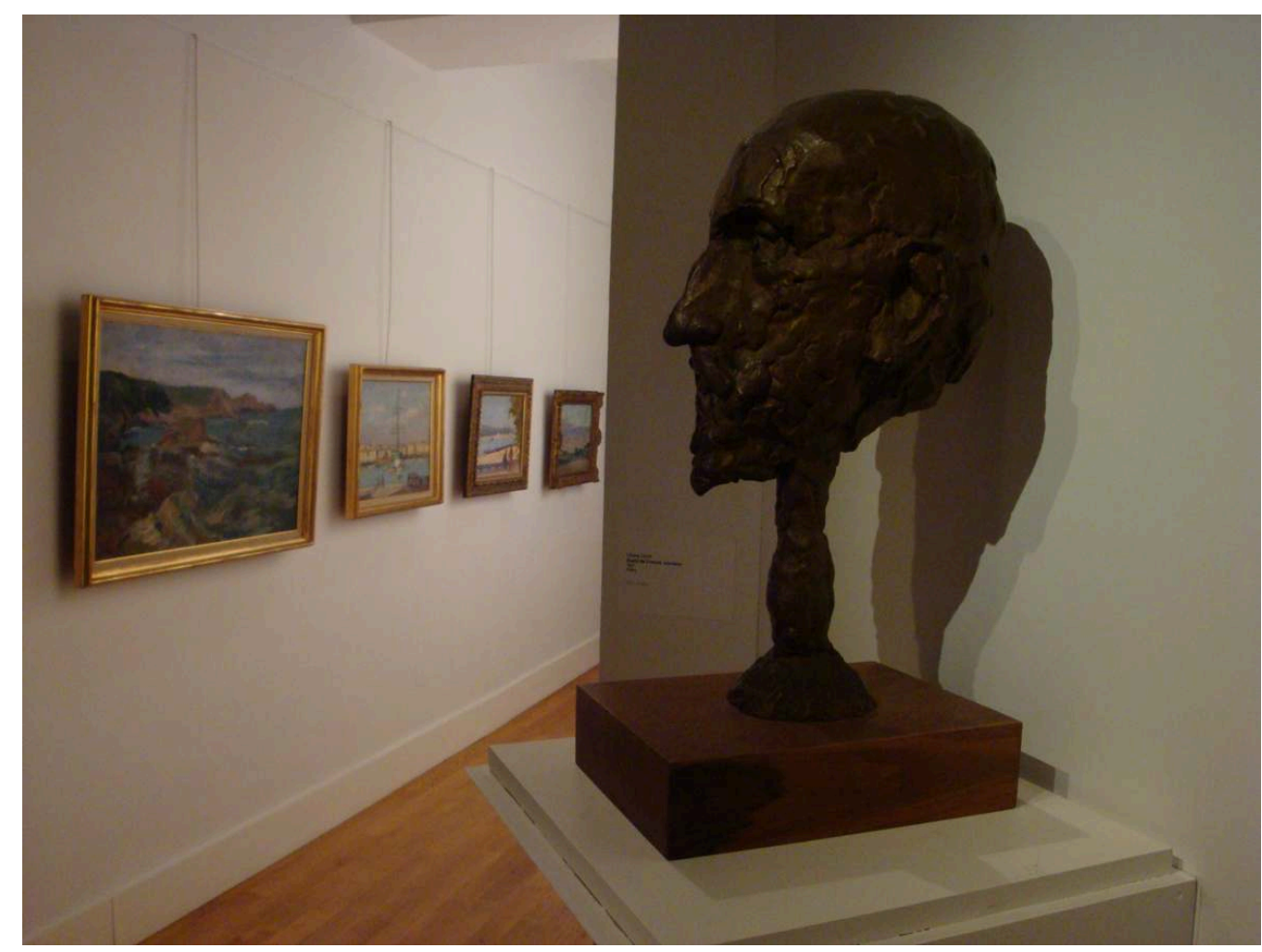

Salle Francis Jourdain - Albert André. Musée d'art et d'histoire, Saint-Denis.

Phot. MAHSD Service des collections. (c) Adagp, Paris, 2016.

4 De 1896 à 1912 il expose régulièrement son travail de peintre, dessinateur ou d'art décoratif, à la galerie Le Barc de Boutteville, à la Société nationale des Beaux-Arts, au Salon des artistes indépendants, à la Libre Esthétique (Bruxelles), à la galerie Druet. Il obtient la médaille d'or à l'Exposition universelle de Paris de 1900 pour les vitraux du théâtre de Loïe Fuller construit par Henri Sauvage. À cette occasion il présente également une maquette d'intérieur ouvrier pour un artisan et sa famille. Il décore la salle à manger de la villa Majorelle à Nancy.

5 Il rencontre Élie Faure ${ }^{2}$ en 1902. En 1912, il s'installe avec sa famille à Esbly, en Seine-etMarne où il ouvre un atelier de menuiserie, Les Ateliers modernes. Il publie avec Léon Werth et Octave Mirbeau une plaquette, plaidoyer pour un nouveau type de mobilier. Il réalise le mobilier de la salle à manger et de la bibliothèque du critique d'art George Besson, dont il fait la connaissance en 1903. Il collabore aux Cahiers d'aujourd'hui, fondé 
par le même George Besson et publie en 1913 le texte de l'architecte viennois Adolf Loos $^{3}$ Ornement et crime. Son orientation professionnelle bascule à partir de cette date. Sans cesser complètement de peindre et d'exposer, il se consacre essentiellement à la création de mobilier et crée le concept des «meubles interchangeables» (fig. 2). Le journal L'Humanité le reconnaît comme l'un des premiers décorateurs français produisant des meubles bon marché qui s'adressent spécifiquement à la classe ouvrière. En 1919 il ouvre à Paris sa boutique au 2 rue de Sèze. Il participe aux salons des artistes décorateurs avec du mobilier, des objets (luminaires, céramiques, tentures, tapis, nappes et verrerie) et répond à de nombreuses commandes, parmi lesquelles on peut citer une Nursery pour Mme James de Rothschild (1920) (fig. 3), le bureau du directeur des magasins de La Samaritaine (1920), collection du musée, la galerie et la maison de campagne de Marius Mermillon, ami de George Besson (1926), la boutique de haute couture Lippé Soeurs (1927), l'appartement de René Gas (1928), le bureau du directeur du Collège de France (1938), encore en place. Il est en 1929 un des membres fondateurs de l'Union des artistes modernes avec Robert Mallet-Stevens, Charlotte Perriand, René Herbst, Pierre Charreau, Hélène Henry..., et participera à leurs divers salons. Il organise en 1932 avec Henri Barbusse et Romain Rolland le Congrès contre la guerre et devient membre de l'Association des écrivains et des artistes révolutionnaires.

\section{Figure 2}

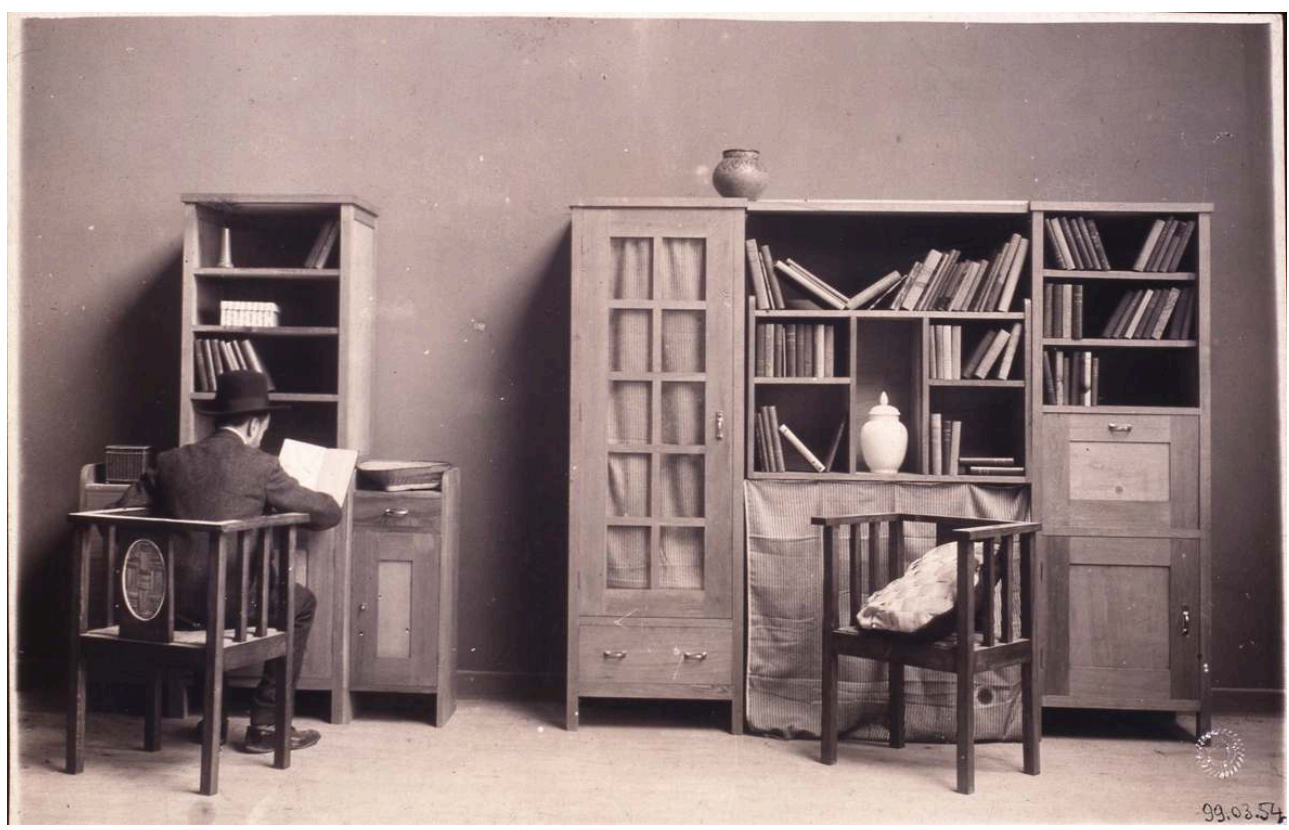

Francis Jourdain, Meubles interchangeables. Musée d'art et d'histoire, Saint-Denis.

Phot. Irène Andréani. (c) Adagp, Paris, 2016. 
Figure 3

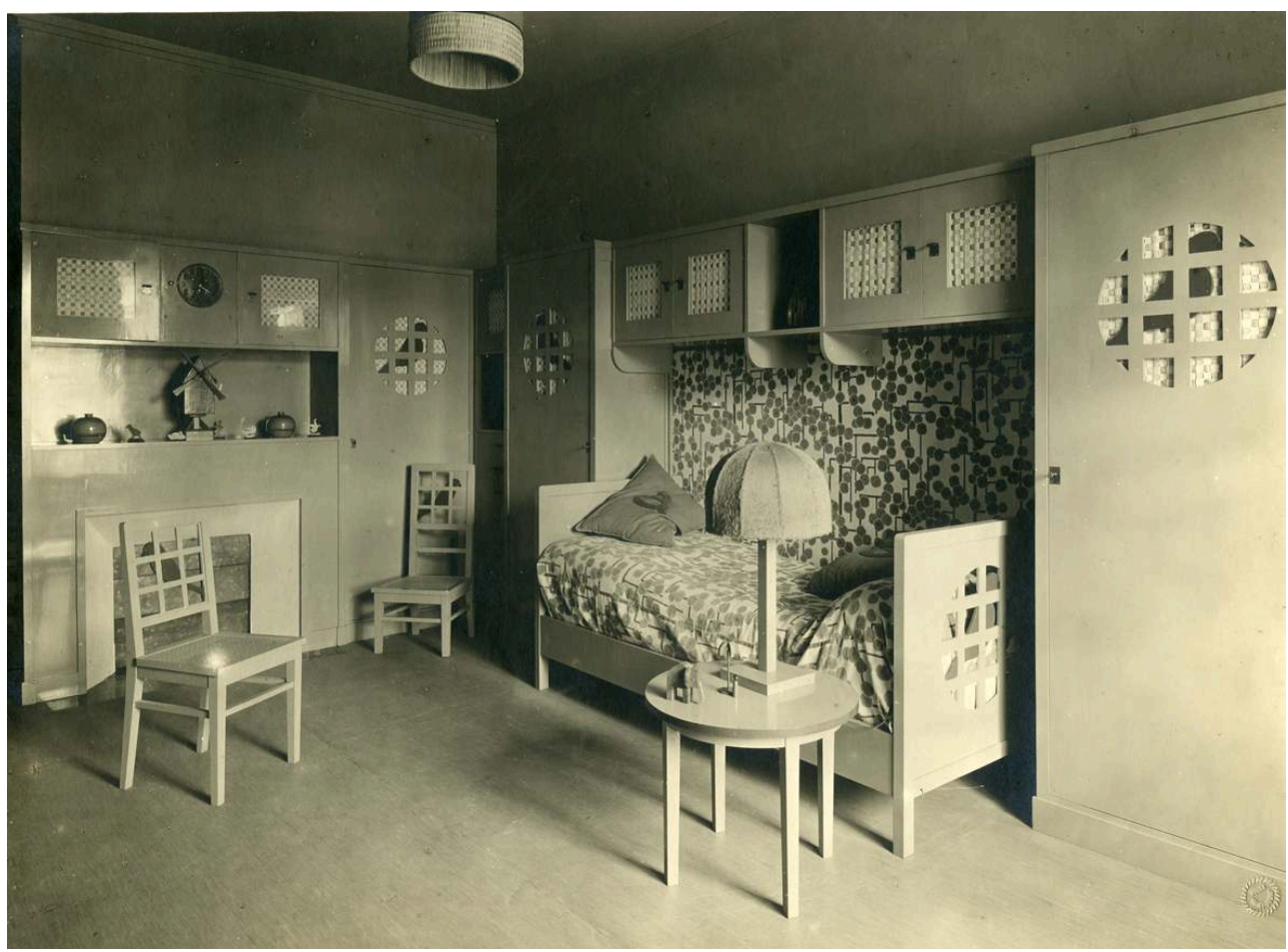

Francis Jourdain, Nursery de Madame de Rothschild (photographie, 1922). Musée d'art et d'histoire, Saint-Denis.

(C) Adagp, Paris, 2016.

Poursuivi par la Gestapo, il se cache et rédige ses souvenirs. Il adhère au Parti communiste français le 5 octobre 1944, le même jour que Pablo Picasso. Après guerre, il se consacre à la critique d'art, rédige des ouvrages et des articles sur les artistes qu'il a connus, sur l'actualité et participe activement à la défense des artistes et des droits humanitaires. Il est le premier président du Secours populaire français. Il décède à Paris le 31 décembre 1958 et est enterré au cimetière Montparnasse le 5 janvier 1959.

\section{La collection du musée}

7 Le musée d'art et d'histoire de Saint-Denis possède une importante collection de l'ensemble du travail de Francis Jourdain. Les premières œuvres entrent dans les collections en 1971 avec la donation George Besson ${ }^{4}$, collectionneur et ami de Francis Jourdain. Il s'agit de peintures, dessins et gravures. Une donation de Lulu Vinès, fille de Francis au nom de l'attachement de son père à la mémoire de la Commune de Paris dont le musée possède un important fonds accessible de manière permanente au public sur plus de $300 \mathrm{~m}^{2}$, intervient en 1974. L'exposition organisée en 1976 par Jean Rollin, conservateur du musée d'art et d'histoire, entraîne d'autres donations en 1977 et 1982, comprenant alors des dessins d'aménagement (sur papier ou sur calque) (fig. 4) et quelques trois cents photographies originales d'aménagement intérieur (fig. 5). D'autres dons de la collection Besson arrivent en 1974, 1980, 1982. Une politique active d'acquisition se développe à partir des années 1990 avec l'aide du Fonds régional d'acquisition des musées et le Conseil général de Seine-Saint-Denis. Le choix se porte sur les objets (céramiques, luminaires) et le mobilier 5 . 
Figure 4

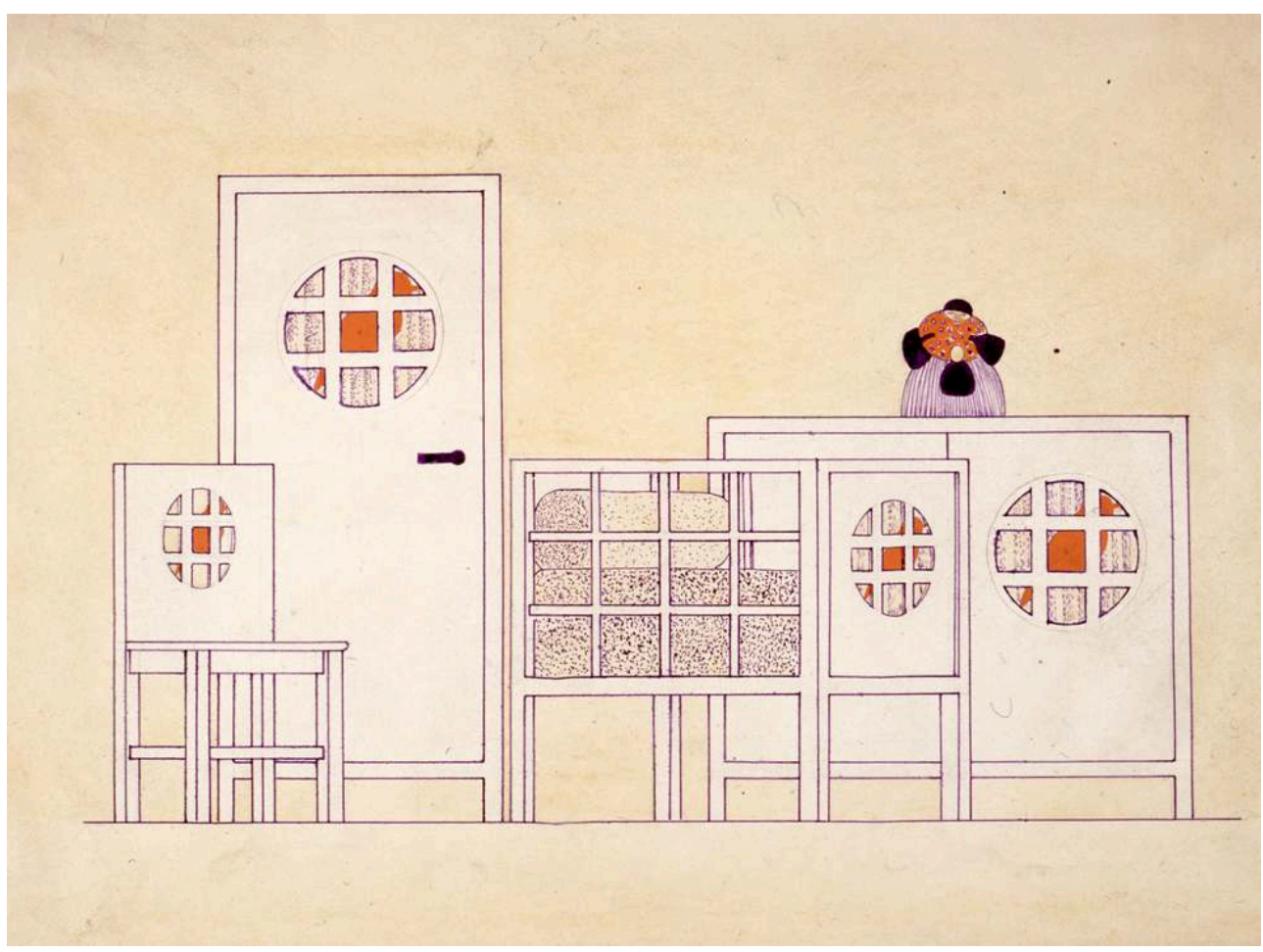

Francis Jourdain, Projet d'aménagement pour la nursery de Madame de Rothschild (dessin sur papier avec collages aux encres de couleur, à la gouache et à l'encre de Chine, 1920). Musée d'art et d'histoire, Saint-Denis.

Phot. Irène Andréani. (c) Adagp, Paris, 2016. 
Figure 5

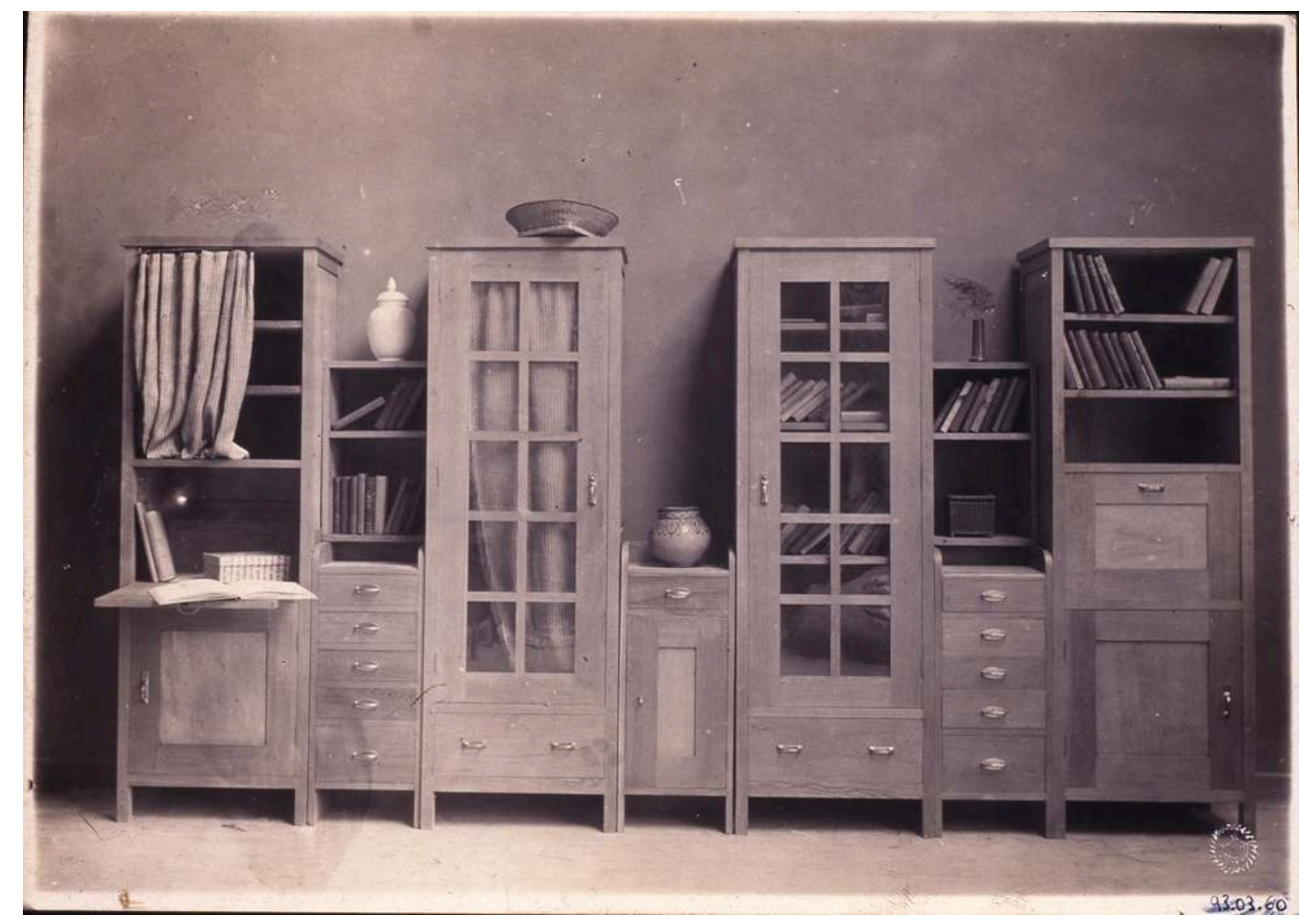

Francis Jourdain, Meubles interchangeables. Musée d'art et d'histoire, Saint-Denis.

Phot. Irène Andréani. (c) Adagp, Paris, 2016.

8 Enfin quatre ensembles entrent dans les collections : en 1985, une salle à manger, don d'un particulier et le bureau du directeur de La Samaritaine (achat), en 1999, le mobilier de l'appartement de George et Jacqueline Besson (donation) et en 2000, achat auprès de la famille du mobilier de la chambre de jeune fille et de la salle de bain l'appartement d'Hélène Henry ${ }^{6}$.

Pour l'appartement des Besson, ce mobilier correspond aux diverses commandes faites au fil des années, la salle à manger en 1910 (boiserie, table et chaises, armoire vitrée, meuble bas) (fig. 6), le bureau en 1912 (aménagement bibliothèque, secrétaire en pitchpin bleu, sofa), le salon en 1920 (sofa, buffet, enfilade, guéridons et fauteuils) et les plafonniers (1922). 


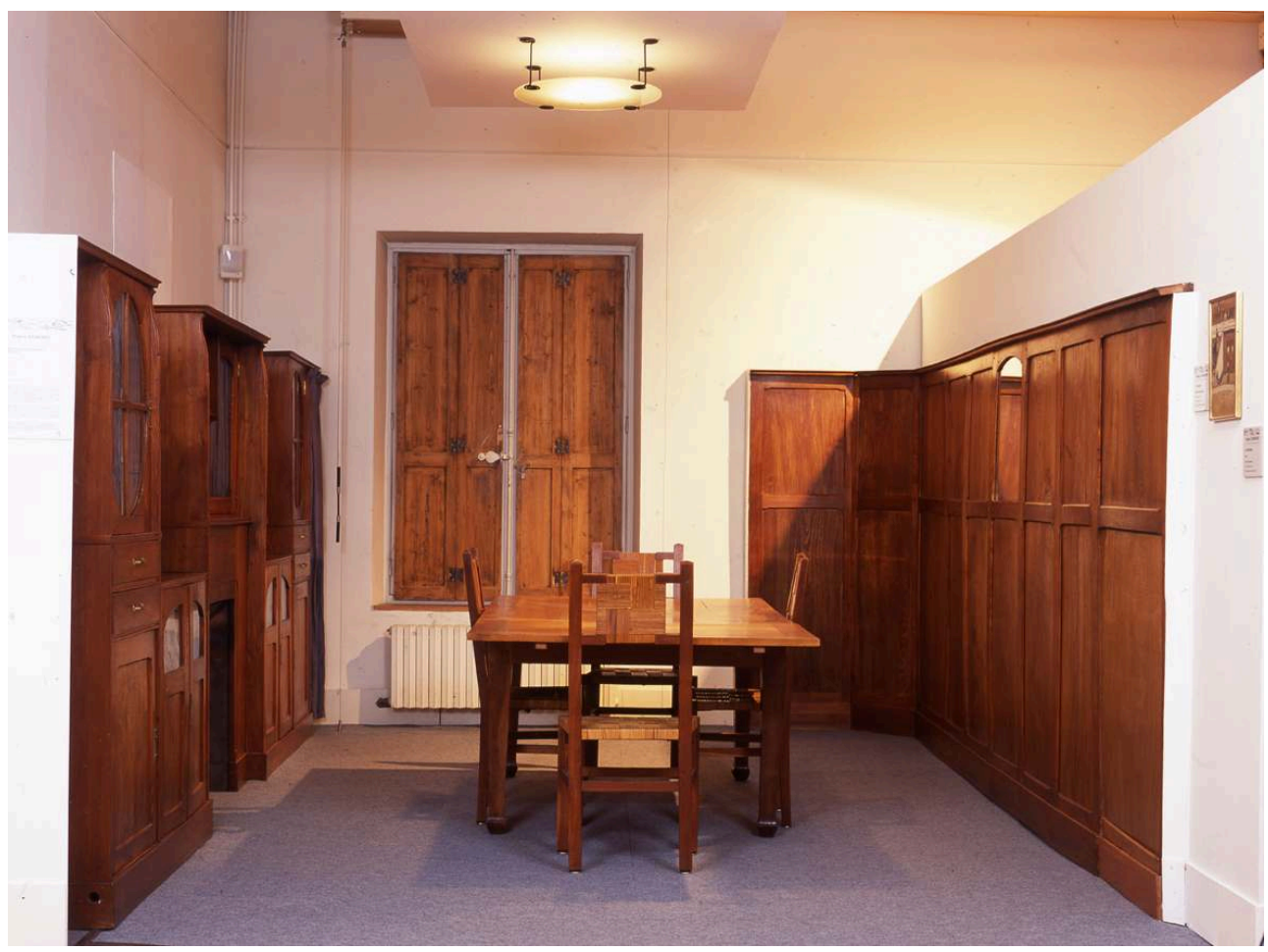

Francis Jourdain, Salle à manger de George Besson. Musée d'art et d'histoire, Saint-Denis.

Phot. Irène Andréani. () Adagp, Paris, 2016.

Des gravures figurent également dans la donation du fonds d'atelier d'Auguste et Eugène Delâtre, auprès de qui Francis a exercé la gravure en couleur suivant la technique dite « à la poupée ».

11 La famille de Francis Jourdain dépose en complément une importante documentation personnelle datant pour la plupart d'après la Seconde guerre. Accessible, elle est consultée régulièrement par les chercheurs.

12 Ainsi le musée d'art et d'histoire, collection publique, possède un ensemble très complet et très large de toutes les facettes du travail de Francis Jourdain. Quelques amateurs avisés et un marchand réputé participent à la diffusion de son œuvre.

En 1992, le département des estampes de la Bibliothèque nationale procède auprès d'un autre descendant, à l'acquisition d'un grand ensemble de documents d'aménagement intérieur, complémentaire de ceux du musée.

Mise en exposition : histoire et actualité, démeubler, un geste architectural vain.

Lorsque le musée d'art et d'histoire rouvre dans les locaux de l'ancien carmel en 1981 et obtient le premier prix européen des musées en 1983, il n'y a pas d'espace d'exposition permanente consacré au fonds Francis Jourdain. La politique active d'enrichissement de ce fonds à partir de 1990 débouche alors sur un grand projet d'exposition organisé dans quatre musées français de mars 2000 à avril 2001.

16 Comment présenter mieux ce projet qu'en citant Françoise Cachin, directeur des Musées de France, dans l'introduction du catalogue : «Quatre musées de province ont permis la redécouverte de Francis Jourdain, conjuguant leurs efforts dans la réalisation 
d'un catalogue et de quatre expositions distinctes insistant, tour à tour, sur un aspect particulier de cet œuvre polymorphe ».

Présentée en premier lieu à Albi, au musée Toulouse-Lautrec, l'exposition est consacrée à Francis Jourdain, peintre, admirateur et ami de Toulouse-Lautrec, évoquant le milieu artistique dans lequel il évolue, au cours de son enfance, de ses années de formation, de ses débuts de peintre (...).

18 L'exposition présentée au musée d'Alès met l'accent sur la personnalité de George Besson, collectionneur et critique d'art. Ami et admirateur de Francis Jourdain, il lui achète des tableaux et lui demande de créer le mobilier de son appartement (...).

19 Troisième étape de l'exposition, le musée d'art et d'histoire de Saint-Denis, qui réunit avec un bonheur paradoxal la mémoire de la Commune et celle du Carmel, est à l'origine du projet. La collection la plus importante et la plus diversifiée d'œuvres de Francis Jourdain y est conservée grâce aux donations successives de la famille Jourdain et de George et Jacqueline Besson et grâce à des acquisitions régulières. L'exposition présente le parcours du créateur et du militant, proche de Zola, de Jaurès, qui, sa vie durant, a lutté contre l'injustice, pour le respect et l'élargissement des droits de l'homme et du citoyen.

L'exposition achève son parcours à Roubaix, au tout nouveau musée d'Art et d'Industrie, dont les collections - peinture moderne et exceptionnel ensemble d'art appliqué à l'industrie - correspondent à l'itinéraire artistique de Francis Jourdain, et dont le mot d'ordre "construire un musée solidaire », un lieu de rassemblement pour tous, rejoint les principes de cet homme de conviction.

21 Aboutissement d'un exemplaire travail en réseau, l'hommage justement rendu à Francis Jourdain permet la mise en lumière de l'œuvre et de la pensée d'un homme moderne qui, conscient très tôt de la nécessité de démocratiser l'art, voulait le beau pour tous? 
Figure 7

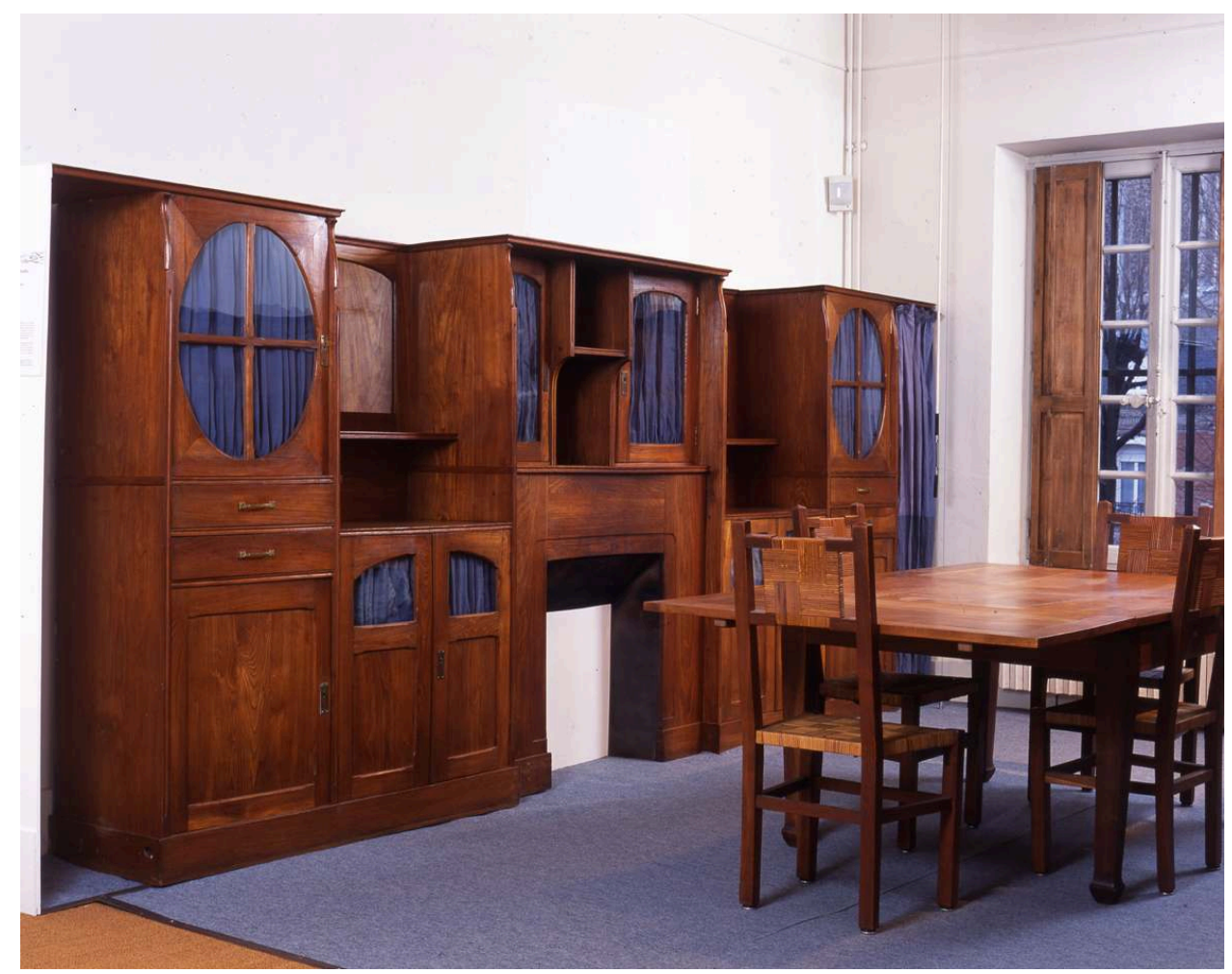

Francis Jourdain, Salle à manger de George Besson. Musée d'art et d'histoire, Saint-Denis.

Phot. Irène Andréani. (c) Adagp, Paris, 2016.

22 Au retour des expositions, il était important alors de trouver une solution de présentation pérenne, lourde charge pour des lieux peu adaptés à une telle présentation. Il fut possible d'associer la présentation des tableaux du peintre Albert André, beau-père de George Besson qui avait fait une donation à l'État avec mise en demeure à Saint-Denis et le mobilier de Francis Jourdain pour le même George Besson. L'espace de la salle, ancien parloir du couvent des carmélites, permettait la mise en situation cohérente, façon "period room ", de la salle à manger de l'appartement du couple Besson (fig. 7) et de la chambre à coucher de l'appartement d'Hélène Henry (fig. 8). Ces deux ensembles, complémentaires, de 1910 et 1927, illustrent parfaitement le concept de « démeublement » que Francis Jourdain met en application à partir des théories de l'architecte viennois Adolf Loos. L'acquisition de l'ensemble d'Hélène Henry avait par ailleurs permis la restauration des fauteuils de George Besson, grâce à la découverte de tissus sous le revêtement récent du sofa du critique d'art, tissu conservé par la famille de la créatrice. 
Figure 8

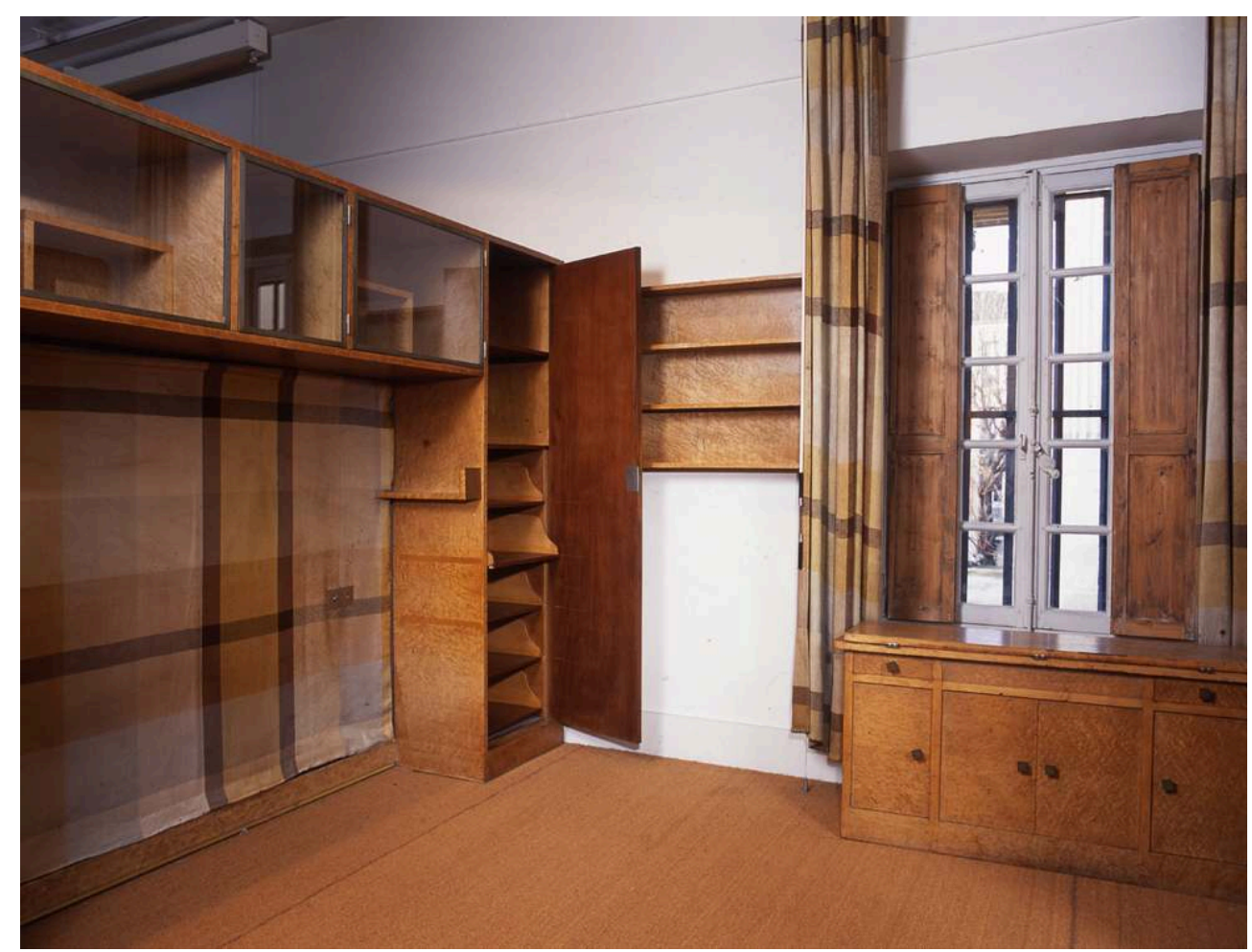

Francis Jourdain, Chambre à coucher d'Hélène Henry. Musée d'art et d'histoire, Saint-Denis.

Phot. Irène Andréani. ( ) Adagp, Paris, 2016.

Ainsi fut-il pendant une dizaine d'années jusqu'à ce que la salle soit entièrement rénovée, le sol changé, l'éclairage refait avec intervention d'un architecte d'intérieur. Geste architectural vain, trahison du principe de Francis Jourdain, où, sous prétexte de mettre en valeur, quelques meubles du salon 1920 de George Besson côtoient une partie de la chambre d'Hélène Henry sur une estrade centrale, dessinant un couloir étroit, mal commode même pour la présentation des peintures (fig. 9). S'il est certain que des vitrines protègent les dessins d'aménagement en regard, les meubles sont posés comme échoués et l'esprit d'ensemble est perdu. Soit, tout est plus beau, tout neuf, mais tourner autour pour voir les tableaux, les meubles, les dessins ne suffit pas. 
Figure 9

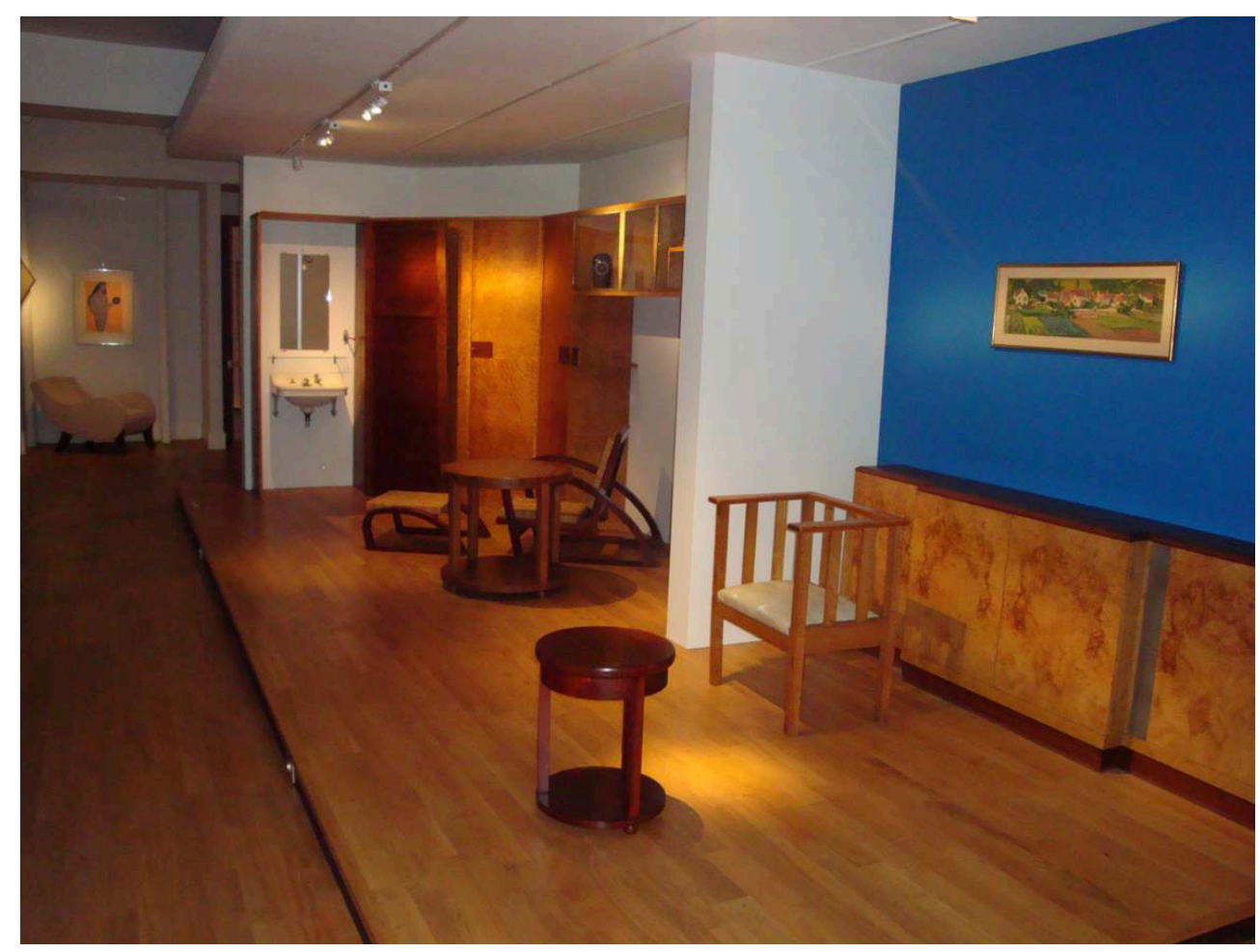

Salle Francis Jourdain - Albert André. Musée d'art et d'histoire, Saint-Denis.

Phot. MAHSD Service des collections. (c) Adagp, Paris, 2016.

Le mobilier de la chambre d'Hélène Henry fut totalement restauré à cette occasion, les canons de serrures manquant refaits avant qu'elle soit, en partie seulement, remontée dans la nouvelle salle rénovée.

Heureusement tout ceci n'est que provisoire, même si ce provisoire dure encore. Un avenir lointain existe, offert à un conservateur futur. Le musée d'art et d'histoire de Saint-Denis non achevé, toute une aile du cloître reste à aménager. On peut donc envisager que l'ensemble important de cet artiste protéiforme, qui épouse les idées de la Commune et rejoint Paul Éluard dans ses convictions et ses combats, qui recoupe le fonds de l'atelier de gravure d'Eugène Delâtre, voie ses espaces d'exposition étendus afin que cet œuvre soit plus amplement magnifié. Plusieurs restitutions pourraient être alors proposées dans les cellules de l'aile est, mettant en regard dessins préparatoires du musée ou de la Bibliothèque nationale, peinture et dessins, tant il est vrai que la couleur que le peintre Jourdain manie avec talent sur la toile, dans ses dessins et ses gravures, rejoint ou répond à son travail d'assemblage des bois, de décor de la céramique (fig. 10). 
Figure 10

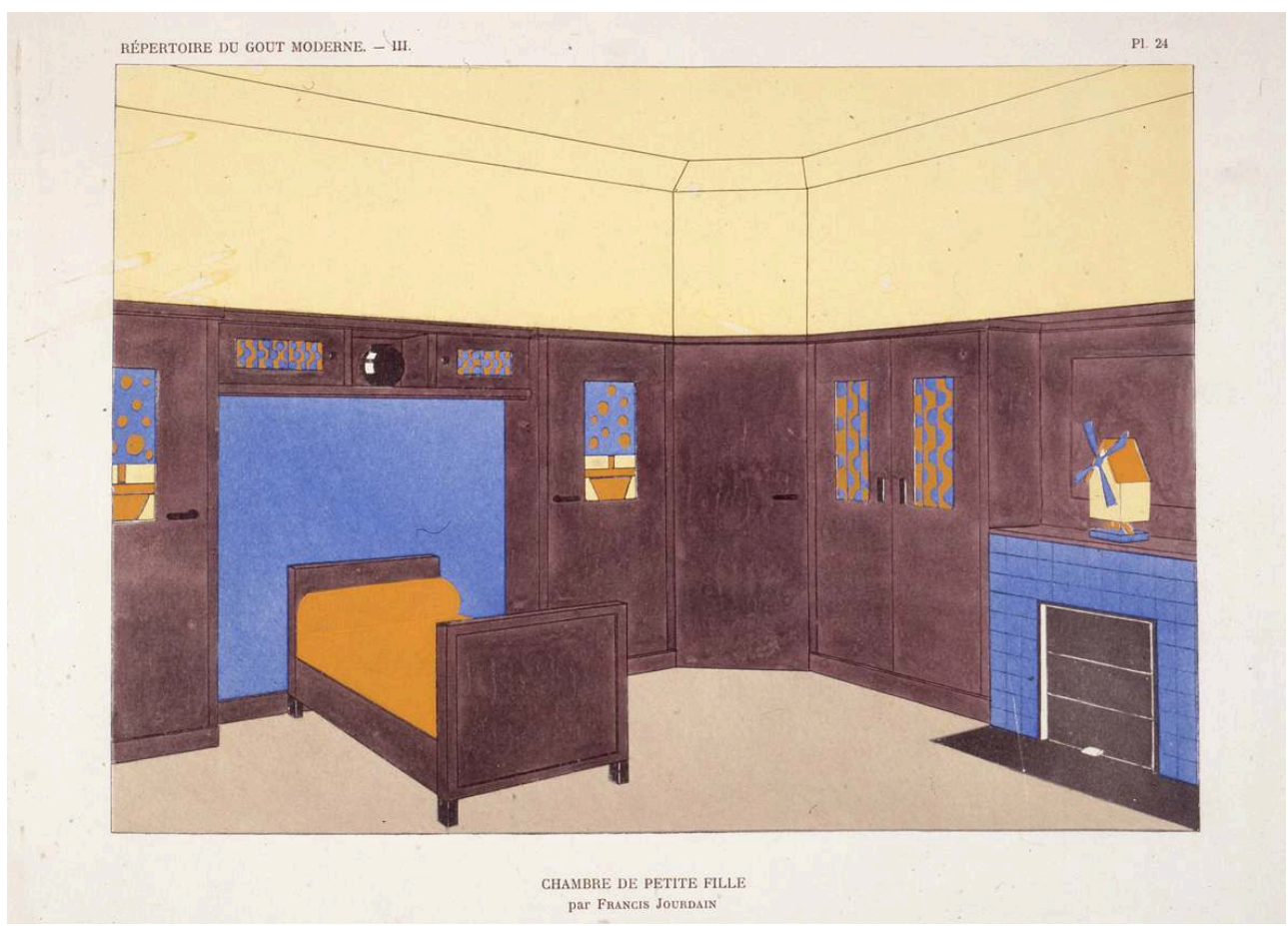

Francis Jourdain, Projet d'aménagement pour une chambre de petite fille (dans Répertoire du goût moderne). Musée d'art et d'histoire, Saint-Denis.

Phot. Irène Andréani. (c) Adagp, Paris, 2016.

Aujourd'hui la pensée et l'œuvre de Francis Jourdain vivent au sein du musée d'art et d'histoire de Saint-Denis, et font partie intégrante du parcours scénographique. Le service des publics organise visites et conférences, participe aux Designer's Days instaurés par le Comité départemental du Tourisme. Une exposition a eu lieu au congrès du Secours populaire de La Villette, en 2005, pour présenter aux adhérents l'œuvre et l'histoire de leur premier président, à la suite de quoi une salle portant son nom a été inaugurée au siège de l'association. Un dépliant spécifique a alors été édité et est encore largement diffusé pour accompagner les prêts d'œuvres.

Aujourd'hui, ce défenseur de «la simplicité comme valeur en soi » et de l'art pour tous reparait de plus en plus sur le devant de la scène, intéresse les chercheurs. Ses œuvres reviennent au goût du jour, circulent dans les expositions et il est heureux que le musée d'art et d'histoire participe à ce renouveau.

\section{NOTES}

1. - Francis Jourdain 1876-1958 Un parcours moderne. Cat. expo. Albi, musée Toulouse-Lautrec, 25 mars au 4 juin 2000 ; Alès, musée-bibliothèque Pierre André Benoît, 24 juin au 3 septembre 2000 ; Saint-Denis musée d'art et d'histoire, 22 septembre au 18 décembre 2000 ; Roubaix, musée d'Art et d'Industrie, janvier à avril 2001, Paris, Somogy 2000. 
2. - Élie Faure (1873-1937), médecin, historien d'art et essayiste français, publie des articles, des ouvrages sur l'art dont une Histoire de l'art en 5 volumes qui reste une référence. Proche de George Besson et de Francis Jourdain, il collabore à la revue Les Cahiers d'aujourd'hui. Depuis 1980, un prix porte son nom qui récompense le meilleur livre d'art de l'année.

3. - Adolf Loos (1870-1933), architecte autrichien, est défenseur du dépouillement intégral en architecture en réaction aux formes qui étaient en vigueur avant lui.

4. - George Besson (1882-1971) est originaire de Saint-Claude (Jura), fils d'un artisan sculpteur en pipe de bruyère. Il devient représentant de l'entreprise familiale. Installé à Paris, il s'enthousiasme pour la peinture que ses revenus lui permettent d'acheter. Il fonde la revue Les Cahiers d'aujourd'hui, publie Les Maitres, monographies des artistes qu'il apprécie et collabore à de nombreuses revues. Grand collectionneur, il fera don de ses magnifiques tableaux de Renoir, Marquet, Signac..., au musée du Louvre, que l'on peut voir aux musées de Besançon, Bagnols-surSèze et Saint-Denis. Voir GONZALEZ, Sylvie. Picasso, Chagall, Signac... Artistes et collectionneur donateurs du musée d'art et d'histoire de Saint-Denis, catalogue de l'exposition, 15 septembre 2006-15 janvier 2007. Saint-Denis : musée d'Art et d'Histoire, 2006.

5. - 1991 : paire de chaises longues et repose pieds, 1994 : boîte céramique, 1995 : paire de fauteuils en hêtre, 1997 : céramique (vase, lampe, service à thé, service à café), lampe métal, applique verre et bois, 1998 : lampe céramique, guéridon en sycomore, paire de fauteuils virgule, 1999: céramique (vases, soliflores au décor inversé vert/crème, pot couvert, bonbonnière, coupe), service à café en porcelaine fine, service à porto, fauteuil prototype (donation), 2003 : céramique, paires d'appliques métal et pâte de verre, 2005 : céramique (donation), lustre fer forgé et pâte de verre.

6. - Hélène Henry (1891-1965), sans formation particulière, ouvre en 1919 un atelier de tissage dont les productions convainquent rapidement les créateurs modernes. En 1920, elle expose pour la première fois au Salon d'automne puis au Salon des artistes décorateurs, soutenue par Francis Jourdain. En 1925, elle est membre de l'Union des Artistes Modernes. Elle sollicite Francis Jourdain pour aménager son appartement encore dans son état d'origine lorsque le musée acquière le mobilier.

7. - Francis Jourdain 1876-1958 Un parcours moderne, Op.cit., p. 7.

\section{RÉSUMÉS}

Le fonds Francis Jourdain du musée d'art et d'histoire de Saint-Denis est l'un des plus importants et les plus diversifiés en France sur l'œuvre de cet artiste aux multiples facettes. Il comprend plusieurs centaines d'œuvres représentatives de l'ensemble de son travail : peintures, dessins, travaux préparatoires pour l'aménagement intérieur et les arts décoratifs, ensembles mobiliers, céramiques et verreries. Peintre, ouvrier d'art, il prône à la suite de l'architecte autrichien Adolphe Loos « la simplicité comme valeur en soi » et l'art pour tous. Il révolutionne le mobilier par le principe, toujours actuel, du meuble interchangeable et ouvre une boutique de vente où ses créations sont distribuées par l'intermédiaire d'un catalogue. Les ensembles sont présentés dans les espaces du musée : la chambre d'Hélène Henry, créatrice de tissus, et le salon de George Besson, collectionneur et critique d'art.

The Musée d'art et d'histoire at Saint-Denis holds an important collection of archives and objects left by Francis Jourdain, an artist of multiple talents and facets. The collection include several 
hundred designs whihc are representative of his work as a whole:paintings, drawings, preparatory sketches for interior designs and decorative arts, furniture ensembles, ceramic and glass pieces. Jourdain was a painter and a skilled art worker and, following in the footsteps of the Austrian architecte Adolphe Loos, he preached simplicity as a value in its own right. He brought revolutionary change to furniture design with the principle of interchangeable pieces of furniture, and opened a shop where his creations could be purchased or ordered by catalogue. His designs are presented in different parts of the museum: the bedroom of Hélène Henry, a textile designer, and the salon of George Besson, an art critic and collector.

\section{INDEX}

Mots-clés : Francis Jourdain, ensemblier, mobilier, art décoratif, catalogue de vente par correspondance, boutique, UAM, Adolphe Loos, architecture d'intérieur, art pour tous, meuble interchangeable

Keywords : Francis Jourdain, furniture designer, decorative arts, mail order catalogue, shop, UAM, Adolphe Loos, interior design, artfopr all, interchangeable piece of furniture

\section{AUTEUR}

\section{SYLVIE GONZALEZ}

Conservatrice en chef du patrimoine, directrice du musée d'art et d'histoire de Saint-Denis sylvie.gonzalez@ville-saint-denis.fr 